\title{
Is the Frequency of Candidemia Increasing in COVID-19 Patients Receiving Corticosteroids?
}

\author{
Cezar V. W. Riche 1,3(1) Renato Cassol ${ }^{2}$ and Alessandro C. Pasqualotto ${ }^{3,4, *}$ \\ 1 Hospital Ernesto Dornelles, Porto Alegre 90160-092, Brazil; cezar.riche@gmail.com \\ 2 Hospital Nossa Senhora da Conceição, Porto Alegre 91350-200, Brazil; renato.cassol@gmail.com \\ 3 School of Medicine, Universidade Federal de Ciências da Saúde de Porto Alegre, Porto Alegre 90050-170, Brazil \\ 4 Santa Casa de Misericordia de Porto Alegre, Porto Alegre 90020-090, Brazil \\ * Correspondence: pasqualotto@santacasa.org.br
}

Received: 22 September 2020; Accepted: 9 November 2020; Published: 13 November 2020

check for updates

\begin{abstract}
Corticosteroids have potent anti-inflammatory and immunosuppressive effects. Recently, these medications have gained importance in the treatment of severe COVID-19. Here we present data demonstrating a marked (10-fold) increase in frequency of candidemia in hospitalized patients with COVID-19 receiving corticosteroids in Brazil. Overall mortality was $72.7 \%$, despite antifungal therapy. Physicians should be aware of the potential risk for candidemia among severely ill COVID-19 patients receiving high-doses of corticosteroids.
\end{abstract}

Keywords: COVID-19; candidemia; steroids

\section{Introduction}

Recent trials have demonstrated the benefit of corticosteroids in the treatment of severe COVID-19 [1-4], which is likely to result in a marked increase in steroids prescriptions in clinical practice. Steroids are known to increase the risks for a variety of infections, but data on serious fungal diseases in critically ill patients receiving corticosteroids for COVID-19 remains scarce. In the Randomized, Embedded, Multifactorial, Adaptive Platform trial for Community-Acquired Pneumonia (REMAP-CAP), despite the benefits observed with hydrocortisone treatment, the authors demonstrated a higher number of serious adverse events in both hydrocortisone groups [2], even though only one patient had documented fungemia in the trial.

The relationship between corticosteroid treatment and fungal infections is well established. Corticosteroids may promote fungal growth in vitro [5]. Moreover, use of corticosteroids have been associated with increased risk for most serious fungal diseases including candidemia, invasive aspergillosis, fusariosis, and mucormycosis [6]. He we present provocative results of a series of candidemia cases following COVID-19, in which the use of steroids was suggested as the main risk factor for fungal infection.

\section{Materials and Methods}

This was a case series of candidemia following COVID-19 in two tertiary care hospitals located in Porto Alegre, Southern Brazil. COVID-19 was diagnosed based on a positive real time PCR test in a patient with appropriate signs and symptoms, according to the criteria established by the Brazilian National as well as the Regional Sanitary Surveillance agencies [7,8]. Candidemia was defined as the presence of one or more Candida species in blood cultures, in patients with temporally clinical manifestations. Patients were included only once in the study. Yeasts were identified at the species level either by Phoenix Automated Microbiology System (Becton Dickinson, Franklin Lakes, NJ, USA) or Vitek-2 System (bioMérieux, Marcy-l'Étoile, France). 
Data were retrospectively collected in the year 2020 between March 16 (date of the first COVID-19 admission in the participant hospitals) and August 31. Data were obtained from infection control records, as well as patients' electronic charts. The Chi-square test with Yates correction was used to compare proportions-WINPEPI v. 11.65 (Abramson, JH) [9], and $p$ values of $\leq 5 \%$ were considered statistically significant.

\section{Results}

Since the beginning of the COVID-19 pandemic, an increase in the frequency of candidemia was observed in two of the hospitals in which authors (CVWR, and RC) were based. During the period of study, incidence of candidemia was 1.43 (hospital 1) and 1.15 (hospital 2) in patients who did not have COVID-19, both per 1000 patients-day. These frequencies were unaltered in comparison to previous year. In contrast, the incidence of candidemia in patients with COVID-19 were 11.83 (hospital 1) and 10.23 (hospital 2) per 1000 patients-day, during the same period ( $p=0.001$ in comparison to the prevalence of candidemia in non-COVID-19 patients). In patients with COVID-19, all cases of candidemia occurred after the use of high-doses of corticosteroids for severe disease. Table 1 summarizes the main clinical findings for these 11 patients. These were mostly male $(n=7 ; 63.6 \%)$, with a median age of 59 years-old (interquartile range, IQR, 49-70 years-old), and a median Charlson score of 4 (IQR, 1-4). Four patients had diabetes, and one was HIV-positive. Ten patients (90.9\%) had central venous catheters in place. These were all non-surgical patients with limited antibiotic exposure, and additional risk factors for candidemia were virtually absent. Candidemia occurred in intensive care for $8 / 11$ patients $(72.7 \%)$, and after a median length of admission to the intensive care unit of 8.7 days. Overall mortality of candidemia following steroid use in COVID-19 was 72.7\% (8/11), while 3/11 patients $(22.3 \%)$ were discharged from the hospital. Overall mortality in COVID-19 patients who did not develop candidemia was 17.7\% (hospital 1) and 22.0\% (hospital 2).

Table 1. Characteristics of patients who had candidemia following corticosteroid use for severe COVID-19.

\begin{tabular}{|c|c|c|c|c|c|c|c|}
\hline $\mathbf{P t}$ & Age/Sex & $\begin{array}{l}\text { Underlying } \\
\text { Conditions }\end{array}$ & $\begin{array}{c}\text { Date of } \\
\text { Admission }\end{array}$ & COVID-19 Management & $\begin{array}{l}\text { ICU Days until } \\
\text { Isolation/Candida } \\
\text { Species }\end{array}$ & $\begin{array}{l}\text { Risk Factors for } \\
\text { Candidemia }\end{array}$ & $\begin{array}{l}\text { Treatment } \\
\text { and } \\
\text { Outcome }\end{array}$ \\
\hline 1 & $44 / \mathrm{M}$ & $\begin{array}{c}\text { DM, } \\
\text { dyslipidemia, } \\
\text { psoriasis, } \\
\text { schizophrenia }\end{array}$ & $\begin{array}{l}13 \text { April } \\
2020\end{array}$ & $\begin{array}{c}\text { Chloroquine } 4 \mathrm{~d} \\
\text { AZI, IVE } \\
\text { MPD } 1 \mathrm{~g} \mathrm{qd} 2 \mathrm{~d} \text {, then } 80 \\
\text { mg bid } 9 \mathrm{~d} \text { and reduction }\end{array}$ & $\begin{array}{l}\text { No ICU admission } \\
\text { C. albicans }\end{array}$ & $\begin{array}{c}\text { DM } \\
\text { CVC } 20 \text { days } \\
\text { PTZ } 5 \text { days } \\
\text { MEM } 8 \text { days }\end{array}$ & $\begin{array}{c}\text { AFG } \\
\text { Discharged }\end{array}$ \\
\hline 2 & $71 / \mathrm{F}$ & $\begin{array}{l}\mathrm{HT} \text {, coronary } \\
\text { artery disease }\end{array}$ & $\begin{array}{l}14 \text { May } \\
2020\end{array}$ & $\begin{array}{c}\text { AZI } \\
\text { MPD } 62.5 \mathrm{mg} \text { bid } \\
2 \mathrm{~d}\end{array}$ & $\begin{array}{l}1 \text { day } \\
\text { C. glabrata }\end{array}$ & $\begin{array}{l}\text { CVC } 7 \text { days } \\
\text { Ceftriaxone } 2 \mathrm{~d} \text {. } \\
\text { PTZ } 7 \text { days }\end{array}$ & $\begin{array}{l}\text { Death } \\
\text { prior to } \\
\text { treatment }\end{array}$ \\
\hline 3 & $69 / \mathrm{F}$ & $\begin{array}{c}\text { DM, HT, } \\
\text { obesity, } \\
\text { cardiac failure, } \\
\text { COPD }\end{array}$ & $\begin{array}{l}16 \text { May } \\
2020\end{array}$ & $\begin{array}{c}\text { HCQ } 1 \text { day }{ }^{\mathrm{a}} / \mathrm{AZI} \\
\text { MPD } 62.5 \mathrm{mg} \text { tid } \\
7 \mathrm{~d}\end{array}$ & $\begin{array}{c}9 \text { days } \\
\text { C. glabrata }\end{array}$ & $\begin{array}{c}\text { DM, } \\
\text { CVC } 9 \text { days } \\
\text { Oxacillin } 4 \text { days } \\
\text { PTZ } 7 \text { days }\end{array}$ & $\begin{array}{l}\text { AFG } \\
\text { Death }\end{array}$ \\
\hline 4 & $37 / \mathrm{F}$ & Asthma & 1 July 2020 & $\begin{array}{c}\text { AZI, IVE } \\
\text { MPD } 62.5 \mathrm{mg} \text { tid } 2 \mathrm{~d} ., \\
\text { then DXM } 6 \mathrm{mg} \text { qd } 3 \mathrm{~d} \text {., } \\
\text { MPD } 62.5 \mathrm{mg} \text { quad } 8 \mathrm{~d}\end{array}$ & $\begin{array}{l}8 \text { days } \\
\text { C. albicans }\end{array}$ & $\begin{array}{l}\text { CVC } 8 \text { days } \\
\text { AMC } 5 \text { days } \\
\text { PTZ } 7 \text { days }\end{array}$ & $\begin{array}{l}\text { AMB-d } \\
\text { FLC } \\
\text { Death }\end{array}$ \\
\hline 5 & $87 / \mathrm{F}$ & $\begin{array}{c}\text { HT, } \\
\text { atrial } \\
\text { fibrillation }\end{array}$ & $\begin{array}{l}10 \text { July } \\
2020\end{array}$ & $\begin{array}{c}\text { AZI, IVE } \\
\text { MPD } 62.5 \text { mg tid } 2 \text { d., bid } \\
2 \text { d., and qd } 3 \mathrm{~d}\end{array}$ & $\begin{array}{l}6 \text { days } \\
\text { C. tropicalis }\end{array}$ & $\begin{array}{l}\text { CVC } 7 \text { days } \\
\text { PTZ } 6 \text { days }\end{array}$ & $\begin{array}{l}\text { AFG } \\
\text { Death }\end{array}$ \\
\hline
\end{tabular}


Table 1. Cont.

\begin{tabular}{|c|c|c|c|c|c|c|c|}
\hline $\mathbf{P t}$ & Age/Sex & $\begin{array}{l}\text { Underlying } \\
\text { Conditions }\end{array}$ & $\begin{array}{c}\text { Date of } \\
\text { Admission }\end{array}$ & COVID-19 Management & $\begin{array}{l}\text { ICU Days until } \\
\text { Isolation/Candida } \\
\text { Species }\end{array}$ & $\begin{array}{l}\text { Risk Factors for } \\
\text { Candidemia }\end{array}$ & $\begin{array}{l}\text { Treatment } \\
\text { and } \\
\text { Outcome }\end{array}$ \\
\hline 6 & $66 / \mathrm{M}$ & HT & $\begin{array}{c}26 \text { July } \\
2020\end{array}$ & $\begin{array}{c}\text { AZI, IVE } \\
\text { MPD } 1 \text { g qd } 2 \text { d., then } 500 \\
\text { mg qd } 1 \text { day, DXM } 6 \text { mg } \\
\text { qd } 9 \text { d }\end{array}$ & $\begin{array}{l}14 \text { days } \\
\text { C. albicans }\end{array}$ & $\begin{array}{l}\text { CVC } 14 \text { days } \\
\text { Cefuroxime } 5 \text { d. } \\
\text { PTZ } 8 \text { days } \\
\text { MEM } 3 \text { days }\end{array}$ & $\begin{array}{c}\text { AFG } \\
\text { Discharged }\end{array}$ \\
\hline 7 & $91 / \mathrm{M}$ & Asthma & $\begin{array}{l}29 \text { July } \\
2020\end{array}$ & $\begin{array}{l}\text { MPD } 250 \mathrm{mg} \text { qd } 1 \mathrm{~d} ., \text { then } \\
\text { DXM } 6 \mathrm{mg} \text { qd } 7 \mathrm{~d}\end{array}$ & $\begin{array}{l}7 \text { days } \\
\text { C. albicans }\end{array}$ & $\begin{array}{l}\text { CVC } 7 \text { days } \\
\text { Amoxicillin } 5 \mathrm{~d} . \\
\text { PTZ } 7 \text { days }\end{array}$ & $\begin{array}{l}\text { AFG } \\
\text { Death }\end{array}$ \\
\hline 8 & $54 / \mathrm{M}$ & $\begin{array}{c}\text { DM, HT, } \\
\text { obesity, } \\
\text { Raynaud } \\
\text { synd., } \\
\text { depression }\end{array}$ & $\begin{array}{l}8 \text { August } \\
2020\end{array}$ & $\begin{array}{c}\text { AZI } \\
\text { DXM } 6 \text { mg qd } 5 \text { d }\end{array}$ & $\begin{array}{l}\text { Day of ICU } \\
\text { admission } \\
\text { C. albicans }\end{array}$ & $\begin{array}{c}\text { DM } \\
\text { AMC } 6 \text { days } \\
\text { PTZ } 2 \text { days }\end{array}$ & $\begin{array}{c}\text { FLC } \\
\text { Discharged }\end{array}$ \\
\hline 9 & $41 / \mathrm{M}$ & $\begin{array}{l}\text { HIV, HCV, } \\
\text { myocardiopathy }\end{array}$ & $\begin{array}{l}15 \text { August } \\
2020\end{array}$ & Prednisone $40 \mathrm{mg}$ qd $4 \mathrm{~d}$ & $\begin{array}{l}\text { Day of ICU } \\
\text { admission } \\
\text { C. albicans }\end{array}$ & $\begin{array}{c}\text { HIV } \\
\text { CVC } 10 \text { days }\end{array}$ & $\begin{array}{c}\text { FLC } \\
\text { Death }\end{array}$ \\
\hline 10 & $57 / \mathrm{M}$ & DM, HT & $\begin{array}{l}23 \text { July } \\
2020^{b}\end{array}$ & $\begin{array}{c}\text { AZI } \\
\text { DXM } 6 \mathrm{mg} \text { qd } \\
10 \mathrm{~d}\end{array}$ & $\begin{array}{l}22 \text { days } \\
\text { C. albicans }\end{array}$ & $\begin{array}{l}\text { DM } \\
\text { CVC } 22 \text { days } \\
\text { AMC } 5 \text { days } \\
\text { PTZ } 8 \text { days }\end{array}$ & $\begin{array}{l}\text { Voriconazole } \\
\text { AFG } \\
\text { Death }\end{array}$ \\
\hline 11 & $59 / \mathrm{M}$ & $\begin{array}{l}\text { HT, chronic } \\
\text { renal disease, } \\
\text { mental } \\
\text { impairment }\end{array}$ & $\begin{array}{l}23 \text { August } \\
2020\end{array}$ & $\begin{array}{l}\text { DXM } 6 \text { mg qd } 3 \text { d., then } \\
\text { Hydrocortisone } 50 \mathrm{mg} \\
\text { qid } 4 \mathrm{~d}\end{array}$ & $\begin{array}{l}3 \text { days } \\
\text { C. albicans }\end{array}$ & $\begin{array}{l}\text { CVC } 4 \text { days } \\
\text { AMC } 4 \text { days }\end{array}$ & $\begin{array}{c}\text { FLC } \\
\text { Death }\end{array}$ \\
\hline
\end{tabular}

Abbreviations: F, female; M, male; Pt, patient; COPD, chronic obstructive pulmonary disease; DM, Diabetes mellitus; HCV, hepatitis C virus; HT, hypertension; AZI, Azithromycin; DMX, dexamethasone; days, d.; HQC, hydroxychloroquine; IVE, Ivermectin; ICU, intensive care unit; MPD, methylprednisolone; AMC, Amoxicillin/clavulanate; AMB-d, Amphotericin B deoxycholate; AFG, Anidulafungin; CVC central venous catheter; FLC, Fluconazole; MEM, Meropenem; PTZ, Piperacillin/tazobactam. ${ }^{\text {a }}$ Hydroxychloroquine was stopped because of cardiac arrythmia. ${ }^{b}$ COVID-19 symptoms began after hospital admission.

\section{Discussion}

In this small case series, we observed a 10-fold increase in the frequency of candidemia in two medical centers in Brazil, in COVID-19 patients taking high doses of steroids. The in-hospital mortality among COVID-19 patients in the study was similar to the published findings of others [10], and even lower than other studies conducted in the same region in Brazil [11]. He observed an impressively high mortality in these patients. It should be noted, however, that our study is limited-mostly by its small sample size, in addition to its retrospective design and its short duration of observation.

Current data suggest low frequency of both bacterial and fungal infections in COVID-19 cases presenting early at the hospital [12]. However, the use of steroids may dramatically change such scenarios. Fungal infections are known to occur after COVID-19, with most studies so far focusing on invasive aspergillosis [13]. In our perspective, the real frequencies and organisms associated with COVID-19 superinfections must be further evaluated and might be increased by immunosuppressive treatments.

In conclusion, steroids are a lifesaving treatment option in COVID-19 pneumonia, but the right dose and timing of such interventions remain to be determined. High corticosteroid doses might be associated with severe superinfections. Physicians should be aware of the potential increase in the frequency of candidemia in patients with COVID-19 treated with corticosteroids.

Author Contributions: Conceptualization, C.V.W.R., A.C.P.; investigation, C.V.W.R., R.C.; writing-original draft preparation, C.V.W.R., A.C.P.; writing-reviewing and editing C.V.W.R., R.C., A.C.P. All authors have read and agreed to the published version of the manuscript.

Funding: This research received no external funding.

Conflicts of Interest: Pasqualotto has received research grants from Gilead, Pfizer, and MSD. He has also given paid talks on behalf of Gilead, Pfizer, MSD, Astellas, United Medical, Teva, and IMMY. 


\section{References}

1. The RECOVERY Collaborative Group; Horby, P.; Lim, W.S.; Emberson, J.R.; Mafham, M.; Bell, J.L.; Linsell, L.; Staplin, N.; Brightling, C.; Ustianowski, A.; et al. Dexamethasone in hospitalized patients with Covid-19-Preliminary report. N. Engl. J. Med. 2020. [CrossRef]

2. The Writing Committee for the REMAP-CAP Investigators; Angus, D.C.; Derde, L.; Al-Beidh, F.; Annane, D.; Arabi, Y.; Beane, A.; Van Bentum-Puijk, W.; Berry, L.; Bhimani, Z.; et al. Effect of hydrocortisone on mortality and organ support in patients with severe COVID-19. JAMA 2020, 324, 1317. [CrossRef] [PubMed]

3. Tomazini, B.M.; Maia, I.S.; Cavalcanti, A.B.; Berwanger, O.; Rosa, R.G.; Veiga, V.C.; Avezum, A.; Lopes, R.D.; Bueno, F.R.; Silva, M.V.A.O.; et al. Effect of Dexamethasone on days alive and ventilator-free in patients with moderate or severe acute respiratory distress syndrome and COVID-19. JAMA 2020, 324, 1307. [CrossRef] [PubMed]

4. Dequin, P.-F.; Heming, N.; Meziani, F.; Plantefève, G.; Voiriot, G.; Badié, J.; François, B.; Aubron, C.; Ricard, J.-D.; Ehrmann, S.; et al. Effect of hydrocortisone on 21-day mortality or respiratory support among critically ill patients with COVID-19. JAMA 2020, 324, 1298. [CrossRef] [PubMed]

5. Ng, T.T.; Robson, G.D.; Denning, D.W. Hydrocortisone-enhanced grow of Aspergillus spp.: Implications for pathogenesis. Microbiology 1994, 140, 2475-2479. [CrossRef] [PubMed]

6. Lionakis, M.S.; Kontoyiannis, D.P. Glucocorticoids and invasive fungal infections. Lancet 2003, 362, $1828-1838$. [CrossRef]

7. Brazilian Health Regulatory Agency-ANVISA. Technical Note GVIMS/GGTES/ANVISA No 07/2020. In Guidelines for Prevention and Epidemiologic Surveillance of SARS-CoV-2 (COVID-19) Infections within Healt Services; Brazilian Health Regulatory Agency_ANVISA: Brasilia, Brazil, 2020.

8. Emergency Operations Center in Public Health-COE/SES-RS. Informative Note 24 COE/SES-RS. In Surveillance of Influenza-like Syndrome and Severe Acute Respiratory Syndrome (SARS) Related to COVID-19 Human Infection, Surveillance Systems, Laboratory Network and Testing Strategies; Emergency Operations Center in Public Health-COE/SES-RS: Porto Alegre, Brazil, 2020.

9. Abramson, J.H. WINPEPI updated: Computer programs for epidemiologists, and their teaching potential. Epidemiol. Perspect. Innov. 2011, 8, 1. [CrossRef] [PubMed]

10. Zhou, F.; Yu, T.; Du, R.; Fan, G.; Liu, Y.; Liu, Z.; Xiang, J.; Wang, Y.; Song, B.; Gu, X.; et al. Clinical course and risk factors for mortality of adult inpatients with COVID-19 in Wuhan, China: A retrospective cohort study. Lancet 2020, 395, 1054-1062. [CrossRef]

11. Baqui, P.; Bica, I.; Marra, V.; Ercole, A.; Van Der Schaar, M. Ethnic and regional variations in hospital mortality from COVID-19 in Brazil: A cross-sectional observational study. Lancet Glob. Health 2020, 8, e1018-e1026. [CrossRef]

12. Hughes, S.; Troise, O.; Donaldson, H.; Mughal, N.; Moore, L. Bacterial and fungal coinfection among hospitalized patients with COVID-19: A retrospective cohort study in a UK secondary-care setting. Clin. Microbiol. Infect. 2020, 26, 1395-1399. [CrossRef] [PubMed]

13. Lansbury, L.; Lim, B.; Baskaran, V.; Lim, W.S. Co-infections in people with COVID-19: A systematic review and meta-analysis. J. Infect. 2020, 81, 266-275. [CrossRef] [PubMed]

Publisher's Note: MDPI stays neutral with regard to jurisdictional claims in published maps and institutional affiliations.

(C) 2020 by the authors. Licensee MDPI, Basel, Switzerland. This article is an open access article distributed under the terms and conditions of the Creative Commons Attribution (CC BY) license (http://creativecommons.org/licenses/by/4.0/). 